\title{
Dynamical limits by downsizing for the photo- induced switching in a molecular material revealed by time-resolved X-ray diffraction
}

\section{Alix Volte}

University Rennes

Celine Mariette

University Rennes

Roman Bertoni

University Rennes

Marco Cammarata

University Rennes

Xu Dong

University Rennes

Elzbieta Trzop

University Rennes

Herve Cailleau

University Rennes

Eric Collet

University Rennes https://orcid.org/0000-0003-0810-7411

Matteo Levantino

European Synchrotron Radiation Facility

Michael Wulff

European Synchrotron Radiation Facility

Jacek Kubicki

University in Poznań

Feng-Lei Yang

Université Paris-Saclay

Marie-Laure Boillot

Université Paris-Saclay

Benoit Corraze

Université de Nantes

Laurentiu Stoleriu

Alexandru Ioan Cuza

Cristian Enachescu 
Alexandru Ioan Cuza

Maciej Lorenc ( $\square$ maciej.Iorenc@univ-rennes1.fr)

University Rennes https://orcid.org/0000-0002-6877-8631

\section{Article}

Keywords: photo-induced phase transition, pump-probe techniques, x-ray powder 17 diffraction, spincrossover molecular materials, cooperativity

Posted Date: May 14th, 2021

DOl: https://doi.org/10.21203/rs.3.rs-515128/v1

License: (9) This work is licensed under a Creative Commons Attribution 4.0 International License. Read Full License

Version of Record: A version of this preprint was published at Communications Physics on June 29th, 2022. See the published version at https://doi.org/10.1038/s42005-022-00940-0. 


\section{Dynamical limits by downsizing for the photo-induced switching in a}

\section{2 molecular material revealed by time-resolved X-ray diffraction}

3 Alix Volte ${ }^{1}$, Celine Mariette ${ }^{1}$, Roman Bertoni ${ }^{1}$, Marco Cammarata $^{1}$, Xu Dong ${ }^{1}$, Elzbieta

4 Trzop $^{1}$, Herve Cailleau ${ }^{1}$, Eric Collet $^{1}$, Matteo Levantino ${ }^{2}$, Michael Wulff ${ }^{2}$, Jacek Kubicki ${ }^{3}$,

$5 \quad$ Feng-Lei Yang ${ }^{4}$, Marie-Laure Boillot ${ }^{4}$, Benoit Corraze ${ }^{5}$, Laurentiu Stoleriu ${ }^{6}$, Cristian

6 Enachescu $^{6}$, Maciej Lorenc ${ }^{1}$

7 IUniv Rennes, CNRS, IPR (Institut de Physique de Rennes) - UMR 6251, F-35000 Rennes, France,

$8{ }^{2}$ European Synchrotron radiation Facility, 71 avenue des Martyrs, F-38000 Grenoble, France,

$9{ }^{3}$ Faculty of Physics, Adam Mickiewicz University in Poznań, Uniwersytetu Poznańskiego 2, 61-614 Poznań,

10 Poland,

$11{ }^{4}$ Institut de Chimie Moléculaire et des Matériaux d'Orsay, Université Paris-Saclay, CNRS, UMR 8182, 15 rue 12 Georges Clémenceau, F-91405 Orsay, France,

13 Institut des Matériaux Jean Rouxel (IMN), Université de Nantes, CNRS, 2 rue de la Houssinière, F-44322 Nantes, 14 France,

15 'Universitatea «Alexandru Ioan Cuza », Bulevardul Carol I nr. 11, Iasi, Romania

16 KEYWORDS: photo-induced phase transition ; pump-probe techniques ; x-ray powder 17 diffraction ; spin-crossover molecular materials ; cooperativity 


\section{Abstract}

2 Cooperative molecular switching at the solid state is exemplified by spin crossover 3 phenomenon in crystals of transition metal complexes. Time-resolved studies with temporal

4 resolutions that separate molecular level dynamics from macroscopic changes, afford clear

5 distinction between the time scales of the different degrees of freedom involved. In this work

6 we use 100 ps X-ray diffraction to follow simultaneously the molecular spin state and the

7 structure of the lattice during the photoinduced low spin to high spin transition in microcrystals

8 of $\left[\mathrm{Fe}^{\mathrm{III}}(3-\mathrm{MeO}-\mathrm{SalEen})_{2}\right] \mathrm{PF}_{6}$. We show the existence of a delay between the crystalline

9 volume increase driven by the propagation of collective volumic strain waves, and the

10 cooperative macroscopic switching of molecular state. Such behaviour is different from the

11 expectation that phase transformation only requires atomic displacements in the unit cell, that

12 can occur simultaneously with propagation of a volumic strain. Model simulations and

13 discussions of the physical picture explain the phenomenon with thermally activated kinetics

14 governed by local energy barriers separating the molecular states. 


\section{Introduction}

2 Dynamical processes induced by a laser pulse in materials are intrinsically multi-scale in time

3 and space. The difference of time scale is significant between the electronic processes, typically

4 occurring within femtoseconds, the coherent atomic displacements ranging from few 10's of

5 femtoseconds to picoseconds reflecting the period of optical phonons, the volume expansion,

6 and finally the slower kinetics dictated by activation energies. It is noteworthy that the

7 macroscopic crystal deformations, such as global volume change, require collective atomic

8 motions driving lattice deformations over long distances, i.e. acoustic wave propagation. The

9 time scale is thus limited by the speed of sound in the crystal. The strain waves are triggered

10 by laser-induced internal stress, generated by ultrafast lattice heating and/or instantaneous electronic change [Thomsen1986, Wright1994]. Recovery of the mechanical equilibrium with the sample environment occurs through wave propagation over the relevant length of the system (crystal size, light penetration depth, ...). The associated acoustic time scale falls in the

14 picosecond range for nanometers, and in the nanosecond range for micrometers in many materials of interest. Moreover, there may exist activation barriers at the local scale, which can further slow down the atomic rearrangements. As opposed to conventional time-averaging experiments, ultrafast time-resolved experiments are typically used to delineate the dynamics of the different degrees of freedom, such as change of electronic distribution, atomic reorganizations, as well as cell deformations [Lorenc2009]. They are key to understanding the non-equilibrium dynamics on material scale, and to harnessing the mechanisms that govern the properties of materials [Bargheer2004, Baum2007, Braun2007, Morrison2014].

The opportunity to photo-induce a phase transition with a laser pulse [Nasu1997, Nasu2004] has opened up a vast field of research ranging from the melting of charge, spin and structural orders in electronically correlated materials [Zhang2014, Basov2017] to molecular switching 
1 in the solid state [Lorenc2009, Cailleau2010, VanderVeen2013a, VanderVeen2013b].

2 Recently, some attention has been turned to phase transitions that explore the possibility of 3 combining the ultra-fast with the ultra-small [Sagar2016, Park2017, Ridier2019]. We

4 previously reported the size effect on an elastically-driven cooperative dynamical response in

5 a switchable molecular crystal, a spin crossover (SCO) iron complex [Bertoni2016a]. Owing

6 to a positive elastic feedback from the expanding lattice on the volume-changing bistable

7 molecules, the number of switched molecules is significantly enhanced and the lifetime of their

8 photo-induced state is prolonged. The time scale of such dynamics showed to be scaling with

9 the size, namely becoming shorter in micro- and nano-crystals than in bulk single-crystals (>

$10100 \mu \mathrm{m})$. This suggested a central role of propagating volume expansion for the cooperative 11 transformation at the scale of a crystallite [Bertoni2016b].

12 At thermal equilibrium, volume change and spin state switching occur together. In contrast, the out-of-equilibrium dynamics triggered by a laser pulse implies a sequence of processes. To establish the respective evolution of the elastic deformation of crystalline volume and the switching of molecular spin state, a simultaneous measurement of the two purportedly coupled parameters is required. To address this challenge, we used time-resolved X-ray diffraction (trXRD) on small crystallites. Our findings were corroborated with extended Monte Carlo simulations.

\section{Experimental Results}

In this study, we focus on an SCO compound [Fe $\left.{ }^{\mathrm{III}}(3-\mathrm{MeO}-\mathrm{SalEen})_{2}\right] \mathrm{PF}_{6}$. The SCO materials serve as prototypical examples of cooperative switching between two molecular electronic 
1 states, Low Spin (LS) and High Spin (HS). The sample was previously identified as suitable

2 candidate for the photo-induced out-of-equilibrium studies in solid state from the angle of

3 elastic properties that lead to strong cooperativity [Bertoni2016a]. The crystalline structure of

4 this material was characterized in previous studies (S1, [Tissot2011, Tissot2012]). At the

5 molecular level, the switching of electronic state of $\mathrm{Fe}^{\mathrm{m}}$ system, from LS ( $\left.\mathrm{S}=1 / 2\right)$ to $\mathrm{HS}$ ( $\mathrm{S}=$

$65 / 2$ ) causes an increase of molecular volume, due to elongation of the Fe-Ligand bonds by

7 around $0,15 \AA$ A between LS and HS states. The molecules in this compound are arranged in a

8 closed packed network [Tissot2011] (figure 1a). It is known that the relative stability between

9 different macroscopic phases in such SCO crystals is ensured by the elastic intermolecular

10 interactions of various strengths, resulting in more or less cooperative transformations

11 [Rat2017, Buron2012]. In the present case, a strongly first order phase transition is observed

around $162 \mathrm{~K}$, with a thermal hysteresis of $3 \mathrm{~K}$, between the low temperature LS phase and the

high temperature HS phase [Tissot2011]. This phase transition is isostructural, as it does not imply any change of symmetry (same space group P-1 and Z=2 for each phase), in a way similar to the gas-liquid transition [Chernyshov2004]. The volume is a totally symmetric parameter and plays central role for an isostructural phase transition. At thermal equilibrium, both the unit cell volume and the concentration of HS molecules show correlated jumps at the phase transition. This is usually observed for two totally symmetric degrees of freedom involved in changes associated with a phase transition without symmetry change [Landau1980].

We performed XRD measurements on a single crystal (see Methods and S2) between $100 \mathrm{~K}$ and $250 \mathrm{~K}$, in order to quantify accurately the volume jump (volume discontinuity at the first order phase transition). The measured temperature evolution of the unit cell volume is displayed in figure $1 \mathrm{~b}$. It shows a significant volume jump of $1.6 \%\left(22 \AA^{3}\right.$ per unit cell) at transition temperature $\mathrm{T}_{\uparrow}=166 \mathrm{~K}$ (both consistent with the values reported in the previous studies [Tissot2011]). Below and above this discontinuity, the thermal expansion is also 
1 significant in both LS and HS phases. The same measurement allowed for accurate

2 determination of the thermal dependence of all six unit cell parameters for each triclinic phase.

3 Their extrapolated evolution can be described with a linear function of temperature $\mathrm{T}$ for both

4 phases (S2). The volume expansion coefficients for HS and LS phases were found to be

5 respectively $0.31 \AA^{3} / \mathrm{K}$ and $0.16 \AA^{3} / \mathrm{K}$ (figure $1 \mathrm{~b}$ ). It is a considerable thermal expansion,

6 leading to volume increase of $33 \AA^{3}$ between 100 and $250 \mathrm{~K}$, in comparison with $22 \AA^{3}$ jump

7 originating from the transition.

\section{Powder XRD study at thermal equilibrium of micro-crystals}

9 In the following, we will discuss measurements performed on micro-crystals of [Fe ${ }^{\mathrm{III}}(3-\mathrm{MeO}-$

10 SalEen) $\left.{ }_{2}\right] \mathrm{PF}_{6}$ embedded in a polymer thin film (see Methods). The small crystallites are plate-

11 shaped, with average dimensions $3.5 \mu \mathrm{m} \times 0.35 \mu \mathrm{m} \times 0.13 \mu \mathrm{m}$ [Tissot2012]. The size is very much dependent on the synthesis conditions. Smaller crystallites are possible to obtain, but these were chosen to ensure diffraction patterns of sufficient quality for a quantitative analysis. The crystallites were dispersed in polyvinylpyrrolidone (PVP) polymer matrix and the composite films were spin-coated on a glass substrate, as described in [Bertoni2012].

The powder XRD measurements were performed at ESRF, ID09 beamline. XRD images were recorded on a $2 \mathrm{D}$ detector in quasi-grazing reflection geometry at $0.2^{\circ}$ incidence angle (see Methods). This experimental geometry was used to reduce the diffuse background due to scattering from the glass substrate and thereby enhance the diffraction from the thin film sample. A typical diffraction image is shown in figure 1c. The diffraction rings from the polycrystalline sample are clearly visible, with a patterning due to preferred orientation of the micro-crystals. Indeed, since the micro-crystals are plate-shaped, they tend to align with the shortest dimension (crystallographic $c$ axis) perpendicular to the film surface. Several microcrystals can also stack in-depth. 
1 Temperature-dependent steady states measurements were first performed to characterize the

2 XRD signatures of the phase transition in the polycrystalline sample. The measured diffraction

3 patterns are shown in figure 1d. Strong diffuse background, mainly attributed to PVP (figure

4 1c) was removed, and the important peak broadening was ascribed mainly to a large X-ray

5 footprint at the small angle incidence. The latter made the analysis challenging, yet as detailed

6 below, the full-pattern refinement was possible. This allowed for retrieval of key parameters,

7 namely the phase fraction and the volume change very accurately.

8 To this end, we applied the Pawley approach [Pawley1981] using the Topas software

9 [Coelho2018], and a similar method to the one recently applied for powder diffraction studies of photo-induced structural changes [Azzolina2019, Mariette2021]. The refinements details are provided in Methods. In short, this method constrains the Bragg peak positions according to the refined unit cell parameters, but allows the Bragg peak intensities to vary freely. At room temperature, the sample is fully in the HS phase. At $100 \mathrm{~K}$, it was not possible to fit the pattern satisfactorily considering LS phase only, therefore a biphasic state had to be considered, in contrast with the bulk single crystal. We ensured the stability of the refinement further by parametrization of the unit cell and peak intensities, as detailed hereafter. Firstly, to describe the volume evolution of both phases as a function of temperature $\mathrm{T}$, the respective six unit cell parameters were forced to follow the thermal expansion determined with the single crystal study and shown in the figure S2. Consequently, the temperature $\mathrm{T}$ was the only parameter required to describe the evolution of all unit cell parameters. Secondly, the relative Bragg peak intensities $I_{h k l}$ were fixed for both phases: $I_{h k l, H S}$ and $I_{h k l, L S}$ were estimated from the refinements of the patterns at room temperature $(\mathrm{RT}=293 \mathrm{~K})$ and $100 \mathrm{~K}$. Thirdly, the complete powder patterns of a biphasic state (LS, HS) were fitted with $\mathrm{X}_{\mathrm{HS}}$ as the only refined parameter. In such a case, $\mathrm{X}_{\mathrm{HS}}$ accounts both for intensity changes and peak shifts, since the thermal expansion is phase-dependent. 
1 The refined $\mathrm{X}_{\mathrm{HS}}$ is plotted in figure if as a function of temperature. A clear change of slope is

2 observed around the transition temperature in the bulk single crystal. Above this temperature,

3 the sample is almost fully in the HS phase. The observed $\mathrm{X}_{\mathrm{HS}}$ evolution correlates very well

4 with the $M * T$ product ( $M$, magnetisation and $T$, temperature) measured by SQUID

5 (Superconducting QUantum Interference Device, see Methods) probing the evolution of the

6 fraction of molecules in the HS state. The spin state conversion in these micro-crystals shows

7 two peculiar features: a gradual conversion (compared to abrupt in bulk), and incomplete

8 conversion at low temperature. The residual $\mathrm{X}_{\mathrm{HS}}$ at $\mathrm{T}=100 \mathrm{~K}$ is equal to $(34+/-3) \%$ is

9 consistent with the previous reports [Bertoni2012]. These two features are explained in terms

10 of size/strain effects and non-homogeneous stress due to surface interaction with polymers,

11 resulting in a broad regime of phase coexistence [Tissot2012, Laisney2020]. The refined $\mathrm{X}_{\mathrm{HS}}$

12 also allows to calculate the average volume:

$V_{\text {average }}(T)=V_{H S}(T) * X_{H S}(T)+V_{L S}(T) *\left(1-X_{H S}(T)\right)(e q .1)$.

14 Concurrence of the gradual spin-state conversion and thermal expansion leads to a very smooth evolution of the average volume. Yet, the average volume $\mathrm{V}_{\text {average }}$ changes slope around $160 \mathrm{~K}$

16 (figure 1e).

17 Thus parametrized powder pattern refinement allows extracting accurate values for both $\mathrm{X}_{\mathrm{HS}}(\mathrm{T})$ and $\mathrm{V}_{\text {average }}(\mathrm{T})$ simultaneously, and the ensuing discussion is hinged upon it. We should nonetheless mention some underlying assumptions. First hypothesis is that the LS and HS phases can be treated as separate diffracting domains. Explicitly, $\mathrm{X}_{\mathrm{HS}}$ quantifies the fraction of ordered HS domains, rather than counting the HS molecules. The excellent correlation between $\mathrm{X}_{\mathrm{HS}}$ and the fraction obtained with SQUID (counting individual molecules) supports our assumption. The second hypothesis is that other structural distortions on temperature within a 
1 given phase have negligible contribution to the changes of $\mathrm{I}_{\mathrm{hkl}}$. This is substantiated with

2 measurements on a single crystal (figure S2) and therefore seems reasonable too.

\section{Time-resolved XRD study of photoinduced dynamics}

4 The tr-XRD images were recorded at $100 \mathrm{~K}$ with the setup described above. The crystallites

5 were excited with 1 ps pump laser at $800 \mathrm{~nm}$, as in previous optical studies [Bertoni2016a].

6 The experimental time resolution was limited by the X-ray pulse duration to $100 \mathrm{ps}$. The details

7 about the setup and data reduction are given in Methods.

8 The time-resolved patterns covering several decades in time are shown in figure 2a. Photo-

9 induced changes can be seen on these patterns for all positive delays, and they are emphasized

10 in the difference patterns (figure $2 b$ ). The comparison with steady state diffraction patterns

11 measured at low and high temperature allows for a qualitative description. The shape of the

12 difference patterns can be explained by a weight transfer of the diffracted intensity from LS-

13 to HS-Bragg peaks. However, the peak shift to smaller q due to volume expansion would

14 produce a similar difference pattern. Therefore, separating these two possible contributions requires more quantitative analysis as described below. A closer inspection of the difference patterns also gives some insight into the dynamics. First changes occur within the $100 \mathrm{ps}$ time resolution. Thereafter, the difference patterns change shape, suggesting a sequence of processes with structurally distinct signatures.

In order to analyze the underlying structural dynamics, the same method of full-pattern refinement as in the temperature study was applied to the tr-XRD patterns. The refinement results are shown in figure $2 \mathrm{c}$. The parametrized model was similar to that used in the temperature study. However, in addition to $\mathrm{X}_{\mathrm{HS}}$, the lattice temperature becomes an adjustable 
1 parameter $\mathrm{T}_{\text {lattice, }}$ to account for the heating and non-equilibrium lattice expansion (see

2 Methods).

3 The time evolution of the structural parameters obtained for the highest excitation density (380

$4 \mu \mathrm{J} / \mathrm{mm}^{2}$ ) reveals multistep dynamics. A small increase of $\Delta \mathrm{X}_{\mathrm{HS}}$ estimated at $7 \%$, accompanied

5 by a small volume increase, is observed at the early step (i.e., within 100 ps time resolution).

6 Even if the initial volume rise cannot be accurately determined due to the 100 ps time

7 resolution, the maximum of volume expansion is sufficiently pronounced and well resolved at

8 around $300 \mathrm{ps}$. At this delay, for high excitation density, the refinement yields a value of $\Delta \mathrm{T}_{\text {lattice }}$

$9=(90+/-5) \mathrm{K}$, corresponding to $\Delta \mathrm{V}_{\text {average }}=19 \AA^{3}$. During this second step, $\Delta \mathrm{X}_{\mathrm{HS}}$ increases

10 very little, even at high excitation density. At such excitation, a more pronounced increase of

$11 \Delta \mathrm{X}_{\mathrm{HS}}$ occurs during a third step on the nanosecond time scale, with a plateau between $\mathrm{t}=20$

$12 \mathrm{~ns}$ and $\mathrm{t}=300 \mathrm{~ns}$, corresponding to the maximum transformation $\Delta \mathrm{X}_{\mathrm{HS}}=23 \%$. Finally, $\Delta \mathrm{X}_{\mathrm{HS}}$

13 and volume decrease simultaneously, and recovery of the values at thermal equilibrium occurs

14 on a sub-millisecond time scale. The most striking observation is the decoupling in time

15 between the dynamics of volume change and $\Delta \mathrm{X}_{\mathrm{HS}}$ : the increase of $\Delta \mathrm{X}_{\mathrm{HS}}$ occurs two orders of magnitude later than the increase of volume. Importantly, their respective dependence on the

17 excitation density is very different. Irrespectively of excitation density, either low or high, the volume expansion exhibits a maximum around $300 \mathrm{ps.} \mathrm{In} \mathrm{contrast,} \Delta \mathrm{X}_{\mathrm{HS}}$ exhibits a continuous decrease in the ns range at low excitation density, and a pronounced increase to a clear maximum at $20 \mathrm{~ns}$ for high excitation density (figure 2c). This observation is in agreement with

21 previous measurements of the $\Delta \mathrm{X}_{\mathrm{HS}}$ by transient optical absorption, and it demonstrates that 22 the nonlinear increase of $\Delta \mathrm{X}_{\mathrm{HS}}$ sets in with a threshold [Bertoni2016a]. However, no significant 23 increase of average volume is observed when $\Delta \mathrm{X}_{\mathrm{HS}}$ reaches its maximum. 
1 The validity of such analysis of non-equilibrium dynamics whereby $I_{\mathrm{hkl}}$ and the lattice

2 expansion are a-priori assumed, can be questioned. Both affect mainly the refined $\mathrm{X}_{\mathrm{HS}}$. In

3 particular, the newly formed HS molecules might be homogeneously distributed within the

4 crystal, rather than organized into separate diffracting phases. This homogeneous distribution

5 would lead to a change of the average structure factor of each phase [Collet2011], that is not

6 taken into account in our analysis. Yet, as already stressed, the evolution of $\Delta \mathrm{X}_{\mathrm{HS}}$ obtained by

7 such analysis is consistent with previous optical measurements (figure 2c) that yield $\Delta \mathrm{X}_{\mathrm{HS}}$

8 directly whether or not there is a phase separation. This very good agreement strengthens the

9 validity of the assumptions made for analyzing the tr-XRD patterns.

10 Monte-Carlo simulations of mechanoelastic model

11 In order to rationalize the experimental results, we applied the mechanoelastic model, also referred to as ball-and-spring model, that had been successfully used in previous studies to simulate the out-of-equilibrium self-amplification [Enachescu2017, Bertoni2019]. In this two14 dimensional model, molecules are mimicked with spheres (balls) whose radii reflect LS or HS state (10\% increase in radius from LS to HS molecules, as observed on the Fe-Ligand bond length). The balls are connected by springs, which replicate elastic interactions occurring in the solid state through the lattice (figure 3a). When molecules (balls) switch, they induce a local deformation of neighboring springs through a change of radii, that will propagate to the adjacent molecules (balls). Hence, the elastic force network accounts for both short- and longrange interactions in the model. The probabilities for switching a given molecule from LS to HS state, and the reverse, depend on the temperature and the local pressure through Arrheniuslike activation. 
1 The elastic stresses originate not only from the molecular swelling upon LS to HS switch, but

2 also from the lattice heating. The latter leads to the so-called thermo-elastic stresses that trigger

3 thermal expansion [Thomsen1986, Matsuda2015, Ruello2015]. It originates from the energy

4 transfer between the photoexcited molecules and the thermal bath of lattice phonons

5 [Lorenc2012]. Because the simulations rely on a harmonic potential, such phenomenon cannot

6 be reproduced by a temperature increase alone, which only modifies the probability of

7 switching. For this work, the Monte-Carlo (MC) model was extended to account for the thermal

8 expansion by allowing distances between molecules to increase without changing the spin state.

9 To factor this aspect of non-equilibrium thermodynamics in MC simulations, the springs were

10 allowed to stretch beyond the HS and LS equilibrium distances at a given temperature (figure

113 b). This mimics thermal lattice expansion. In practice, we started with a simulation assuming

12 a homogeneous increase of temperature, with $15 \%$ increase for HS and LS spring lengths.

13 Following this very first MC step, the spring lengths recover equilibrium positions

14 exponentially, with the same time constant as the recovery of equilibrium temperature. Such

15 extension of the insofar used model is a very simple yet satisfactory approximation that allows independent treatment of volume change and spin transition.

\section{Discussion on the physical picture of photo-induced multistep dynamics}

18 We now consider the implications of the observed multistep structural dynamics and propose 19 a comprehensive scheme for the macroscopic photo-switching. In particular, we discuss the 20 origin of a significant delay between the volume expansion process and the transformation 21 from LS to HS phase.

22 The initial step of photo-excitation by a 100 fs laser pulse was described previously

23 [Camarata2014]. Direct photo-excitation from LS to HS molecular state is forbidden, so the 
$1800 \mathrm{~nm}$ light is used to photo-excite a LS molecule to a short-lived intermediate singlet state,

2 followed by an ultrafast intersystem crossing towards the HS state [Hauser1986]. The latter is

3 structurally relaxed on the ps time scale. The switched molecules are uniformly distributed in

4 each microcrystal, since the thickness of microcrystals is much smaller than the penetration

5 depth of laser light (approximately $5 \mu \mathrm{m}$ at $800 \mathrm{~nm}$ [Bertoni2016a]). Initial $\Delta X_{\mathrm{HS}}$ is small and

6 it scales linearly with the excitation density [Bertoni2016a], here estimated at $7 \%$ for the high

7 excitation density (figure $2 \mathrm{c}$ ). Importantly, the absorbed photon energy $(1.55 \mathrm{eV}$ ) is much

8 higher than the energy difference between HS and LS ground states (tens of meV). In

9 consequence, most of the absorbed energy is dissipated through lattice heating. Complementary

10 time resolved mid-IR measurements (S3) to monitor the vibrational population of nascent HS

11 molecules show that it is reasonable to assume that the transfer of heat to the lattice is complete

12 within few ps.

13 During this initial process at the molecular scale, the volume is constrained, and uniformly

14 distributed stresses are generated through both the swelling of switched molecules and the

15 lattice heating. Subsequently, volume starts to expand through a coherent propagative process

16 occurring at the microcrystal scale. Strain waves are launched directly from the surface of

17 microcrystals to recover mechanical equilibrium with the environment, which is reached when

18 the internal and the atmospheric pressure become equal [Thomsen1986, Wright1994,

19 Ruello2015]. Such strain wave picture is supported by the observation of a maximum of the volume expansion occurring at $300 \mathrm{ps}$, irrespective of the excitation density. It corresponds to the travel time of the volumic deformation through a microcrystal [Schick2014]. Thus, taking a sound velocity $\mathrm{v} \sim 4 * 10^{3} \mathrm{~m} \cdot \mathrm{s}^{-1}$ estimated from previous measurements on similar samples [Parpiiev2017] and $\mathrm{L} \sim 1.3 \mu \mathrm{m}$ the average crystal dimension, the associated acoustic time scale is $\tau=\mathrm{L} / \mathrm{v}=1.3 * 10^{-6} / 4^{*} 10^{3}=330 \mathrm{ps}$. Moreover, the initial photo-switching of $7 \%$ of the molecules causes the average volume jump of only $1.5 \AA^{3}$ (figure 1e), significantly smaller 
1 than the observed volume change of $19 \AA^{3}$ at the maximum of volume expansion(figure $2 \mathrm{c}$ ). It

2 indicates that the lattice heating is dominant in this process. The estimated temperature rise at

3 the surface due to imparted laser energy $(\mathrm{S} 4), \Delta \mathrm{T}_{\text {lattice }}=105 \mathrm{~K}$, corroborates the experimentally

4 found $\Delta \mathrm{T}_{\text {lattice }}=90 \mathrm{~K}$. After $300 \mathrm{ps}, \Delta \mathrm{V}_{\text {average }}$ decreases continually, due to heat transfer to the

5 polymeric environment.

6 Even for the high excitation density, no significant increase of $\Delta X_{\mathrm{HS}}$ is observed during the 7 acoustic step. It contrasts with $\Delta \mathrm{X}_{\mathrm{HS}}=23 \%$ on the $10-100 \mathrm{~ns}$ time scale, in agreement with 8 previous optical measurements (figure 2c). A clear decoupling in time between the dynamics 9 of volume expansion and molecular switching may be explained by a characteristic feature of 10 SCO compounds, namely the existence of energy barriers between LS and HS state at 11 molecular scale. They activate slower kinetics determined by Arrhenius-like probabilities, as introduced in the mechanoelastic model. Our simulations suggest that the delay of the activation is indeed independent of the size of the simulation box (S5.2), unlike the time taken by the strain wave to travel through a microcrystal. At $300 \mathrm{ps}$, the molecular state is not equilibrated with the lattice thermal bath, and remains essentially frozen by local energy barriers. The volume expansion redistributes pressure in a way that favors HS over LS (S5.1), 17 yet it does not cancel the effect of the energy barrier between these two states and the transformation can only occur on longer time scale. Moreover, the occurrence of the second increase of $\Delta \mathrm{X}_{\mathrm{HS}}$ depends in a non-linear fashion on the excitation density. No such increase is observed at low excitation (figure 2c), in accordance with the threshold effect evidenced by optical measurements. It is a manifestation of cooperative response during the phase transformation: intermolecular interactions give rise to self-amplification by internal feedback. By contrast, a linear elastic response for the propagating volume is observed. The delay by 24 almost two temporal decades between the two processes is relatively long. It is fair to assume that achieving a phase transition at the macroscopic scale requires more time than does the 
1 switching of independent molecules [Boukheddaden2000]. Also, it is noteworthy that no

2 additional volume increase is observed when $\Delta \mathrm{X}_{\mathrm{HS}}$ reaches maximum. It is possible that part

3 of the thermal energy stored in the lattice is spent on phase transformation. Consequently, the

4 lattice temperature and volume would decrease and counterweight the increase of volume

5 originating from phase transformation.

6 Finally, after this step which occurs only at high excitation density (figures $2 \mathrm{~b}$ and $3 \mathrm{c}$ ), $\Delta \mathrm{V}_{\text {average }}$

7 and $\Delta \mathrm{X}_{\mathrm{HS}}$ relax simultaneously on $10 \mu \mathrm{s}$ time scale (50\% decrease) to recover equilibrium with

8 the environment. This is about 2 decades longer compared to a faster recovery under the effect

9 of the low excitation, below the threshold. It is hence justified to conclude that the phase

10 transformation associated with a sufficiently expanded volume prolongs the lifetime of the

11 transient high spin state.

12 The complete photo-induced cycle is drawn schematically in figure 4. It delineates time scales

13 for multiscale dynamics triggered by a laser pulse at a molecular level and on the macroscopic

14 scale. It emphasizes that the mechanisms involved in spin state switching through the excited state and the ground state are different. The photophysics of the excited state was largely covered in the literature, yet the cycle at the macroscopic level barely starts there. The figure depicts temporal decoupling at the macroscopic scale between the fast collective dynamics of volume dilation and the slow thermally activated kinetics of the LS-to-HS transformation.

\section{Conclusion}

20 First and foremost, these results demonstrate the capacity of time-resolved X-ray powder diffraction to simultaneously determine the photoinduced dynamics of average unit cell parameters and phase fraction in a molecular switchable material. A strongly discriminating quantitative structural analysis [Freyer2013, Mariette2021] allowed to establish 
1 unambiguously a sequence of events by which photo-induced spin state switching occurs from

2 molecular to material scale. In particular, the experimental results support a scheme for the

3 multiscale dynamics at a macroscopic scale whereby the volume expansion and the increase of

4 switching molecules are clearly separated in time. The time scale of the dynamics of volume

5 expansion agrees with a propagative picture of collective atomic motions such as elastic waves,

6 while the cooperative self-amplification upon phase transformation takes place later. A model

7 simulation accounting for thermally activated kinetics, governed by local energy barriers

8 separating LS and HS molecular state, reliably reproduced all important experimental features

9 (figure $3 \mathrm{a}$ and $3 \mathrm{~b}$ ). The situation is fundamentally different from that encountered in systems

10 with no local energy barriers. In such a case, the transformation implies only ultrafast structural

11 dynamics of atomic displacements on the optical phonon time scale. Those dynamics follow

12 the propagation of volume expansion as observed recently in another isostructural

13 transformation [Mariette2021]. In the case of SCO, the volume must be sufficiently expanded

14 to promote the thermally activated transformation towards the HS phase at the macroscopic

15 scale. Should energy barrier be very low, the macroscopic transformation would be limited by the propagation of volumic strain wave on the acoustic time scale.

17 In conjunction with the earlier optical studies that revealed size dependent switching time [Bertoni2016a], and a priori expectation that in the ever so smaller nanoparticles the macroscopic switching of the molecular state can be indefinitely fast, the new results point to a bottleneck in the switching dynamics. Hence, our findings define the ultimate time scale for macroscopic transformation of molecular state in nanoscale objects for this class of solids.

They do not obey the rule "the smaller the faster" [Ridier2019]. This finding could motivate an optimised material design, scalable with size dependent dynamics and intrinsic energetics.

24 A new perspective for the design of functional spin-crossover nano systems could reside in chemical leverage of the energy barriers by tuning the ligand field, or other methods. 


\section{METHODS}

\section{Single crystal X-ray diffraction}

3 Temperature-dependent $\mathrm{X}$-ray diffraction (XRD) study was performed on [Fe ${ }^{\mathrm{III}}(3-\mathrm{MeO}-$

4 SalEen) $\left.{ }_{2}\right] \mathrm{PF}_{6}$ single crystal using a laboratory SuperNova Agilent Technologies 4-circle

5 diffractometer, with $\mathrm{Cu}-\mathrm{K}_{\alpha}$ microsource $(\lambda=1.5406 \AA)$ and EosS2 CCD camera. The sample

6 was cooled down using 800 Plus nitrogen flow cryostat from Oxford Cryosystems. To obtain

7 good precision of cell parameter change with temperature, a set of short scans within $2 \AA$

8 resolution was taken from $100 \mathrm{~K}$ to $250 \mathrm{~K}$, with 2 to $5 \mathrm{~K}$ steps. Data reduction was performed

9 with CrysAlisPro software [CrysAlisPro2018]. The single crystal had a plate shape, with a long

10 dimension around $100 \mu \mathrm{m}$.

11 Samples

12 The Fe $\mathrm{FII}^{\mathrm{III}}$ compound was prepared in the form of a powder of pure microcrystals as previously 13 reported [Tissot2012]. The microcrystals (typical dimensions of $3.5 \mu \mathrm{m}, 0.35 \mu \mathrm{m}, 0.13 \mu \mathrm{m}$

14 from TEM) were processed in polyvinylpyrrolidone (PVP, $\mathrm{MM}=45000 \mathrm{gmol}^{-1}$ ) thin films

15 formed by spin-coating on glass substrate [Bertoni2012].

16 X-ray diffraction on polycrystalline sample

\section{Experimental setup}

18 X-ray diffraction experiment was performed at ESRF, ID09 beamline during hybrid mode (7/8 th continuous filling +1 isolated electron bunch). The isolated electron bunch was used in the experiment. The ID09 setup was discussed in detail previously [Cammarata2009]. Briefly, fast rotating choppers were used to isolate X-ray single pulses (each $100 \mathrm{ps}$ long) at $40 \mathrm{~Hz}$ repetition 
1 rate. The X-rays energy was centered at $18 \mathrm{keV}(\lambda=0.6888 \AA)$ with $1.5 \%$ bandwidth. The

2 beam size at sample position was 0.1 (horizontal) x 0.02 (vertical). Diffracted X-rays were

3 integrated on a Rayonix MX170-HS CCD detector. Each image was recorded with 1000 shots

4 exposure.

5 For the thermal study at equilibrium, sample was cooled down using a nitrogen cryostream

6 700Plus from Oxford Cryosystems and diffraction images were measured with $1 \mathrm{~K}$ steps from

$7293 \mathrm{~K}$ to $100 \mathrm{~K}$.

8 For the time-resolved study, a synchronized laser $(800 \mathrm{~nm})$ at $40 \mathrm{~Hz}$ was used to excite the 9 sample with $11^{\circ}$ incident angle. Laser Beam size was $1.2 \times 0.23 \mathrm{~mm}^{2}$, leading to a laser 10 footprint on sample of $(6.3 \times 0.23) \mathrm{mm}^{2}$. The sample was cooled down to $100 \mathrm{~K}$. Diffraction 11 patterns were measured with $60 \mu \mathrm{J} / \mathrm{mm}^{2}$ and $380 \mu \mathrm{J} / \mathrm{mm}^{2}$ laser excitation, for time delays in 12 the following lists, respectively: [-50 ps, 0 ps, 50 ps, 100 ps, 300 ps, 1 ns, 3 ns, $10 \mathrm{~ns}, 30 \mathrm{~ns}, 100 \mathrm{~ns}, 300 \mathrm{~ns}, 1 \mu \mathrm{s}, 3 \mu \mathrm{s}, 10 \mu \mathrm{s}$, $100 \mu \mathrm{s}, 300 \mu \mathrm{s}, 1 \mathrm{~ms}]$

[-50 ps, 0 ps, 50 ps, 100 ps, 200 ps, 300 ps, 700 ps, 1 ns, $1.5 \mathrm{~ns}, 3 \mathrm{~ns}, 5 \mathrm{~ns}, 7 \mathrm{~ns}, 10 \mathrm{~ns}, 30 \mathrm{~ns}$, $100 \mathrm{~ns}, 300 \mathrm{~ns}, 1 \mu \mathrm{s}, 3 \mu \mathrm{s}, 10 \mu \mathrm{s}, 30 \mu \mathrm{s}, 100 \mu \mathrm{s}, 300 \mu \mathrm{s}, 1 \mathrm{~ms}]$

17 Measurements were performed with shuffled delays, to detect any drift effects during the experimental time.

\section{Data Reduction}

20 The diffraction images were azimuthally integrated using pyFAI [Ashiotis2015]. Background was subtracted using scikit-ued python library [Rene2017]. Negative reference patterns (-5 ns) were used to calculate differential patterns and averaging with trx python library 


\section{Powder patterns refinement}

2 Full powder pattern refinements were performed after the data reduction step described above.

3 Peak profiles and sample displacement were described from a fundamental parameter approach

4 using expressions derived in [Rowles2017] with the TOPAS software [Coelho2018]. The

5 incident wavelength was fixed $\lambda=0.6888 \AA(\mathrm{E}=18 \mathrm{keV})$ with $\Delta \mathrm{E} / \mathrm{E}=1.5 \%$ (FWHM

6 gaussian). The beam height at sample position was fixed to $20 \mu \mathrm{m}$. Sample displacement was

7 refined once and fixed for a given temperature / time scan. $\alpha_{i}$ was set to $0.2^{\circ}$ for all scans, and

8 refined value remained within $0.2^{\circ}+/-0.05^{\circ}$ interval. Background subtracted patterns were

9 refined on [0.3 - 1.9] $\AA^{-1} \mathrm{q}$ range. Typical $\mathrm{R}_{\mathrm{wp}}$ was $(19+/-2) \%$.

10 Magnetic susceptibility

11 Magnetic measurements were carried out using a Quantum Design SQUID magnetometer

12 (MPMS5S Model) calibrated against a standard palladium sample. Data were collected

13 between 250 and $50 \mathrm{~K}$, at a rate of $0.67 \mathrm{~K} / \mathrm{min}$ with a small number of microcrystals

14 embedded in PVP, once removed from the glass substrate. 
a)

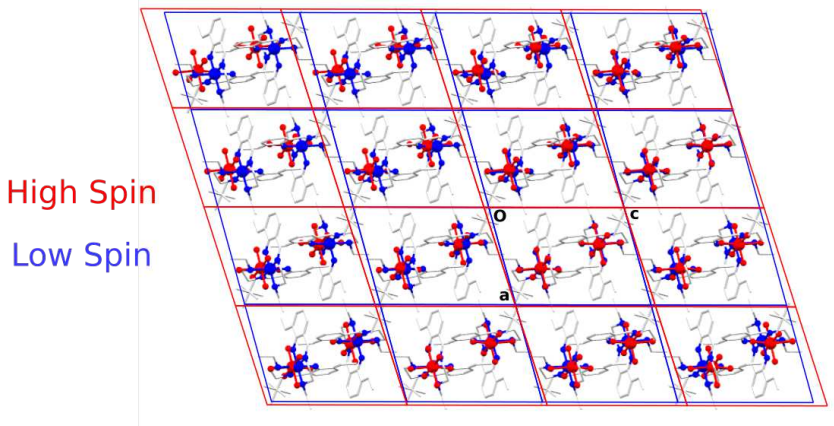

ID09, ESRF, micro-crystals thin film

c)

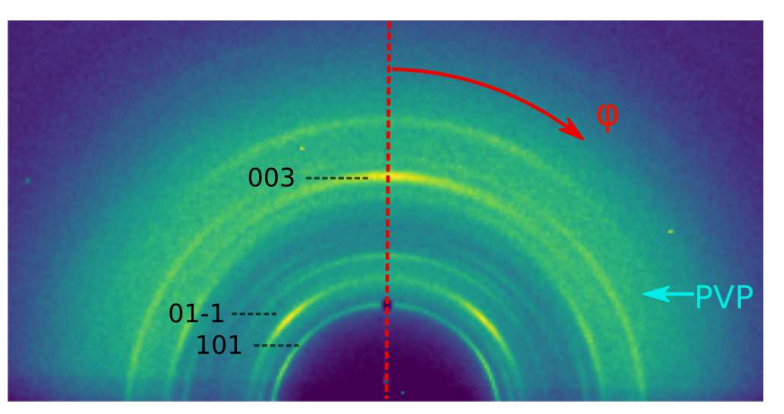

d)

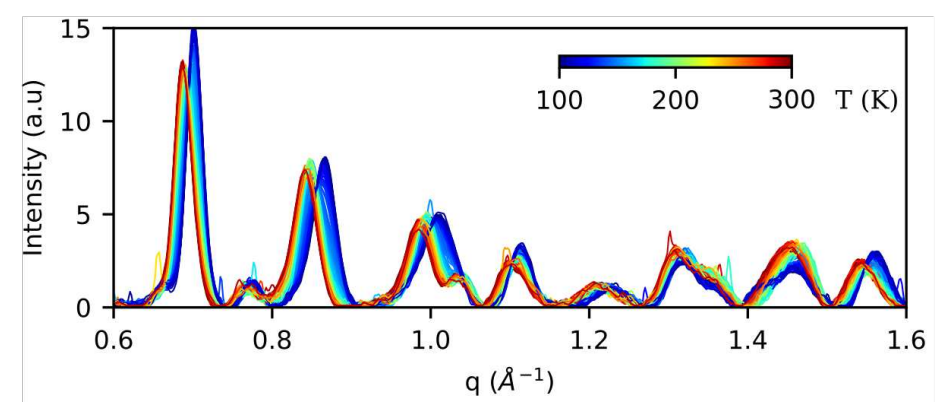

b)

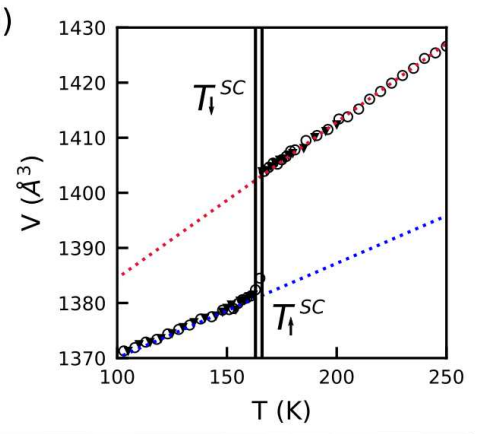

3 Figure 1. a) $\left[\mathrm{Fe}^{\mathrm{m}}(3-\mathrm{MeO}-\mathrm{SalEen})_{2}\right] \mathrm{PF}_{6}$ molecular lattices in LS (100 K, blue) and $\mathrm{HS}$ (293 K,

4 red) state, shown in the $(\boldsymbol{a}, \boldsymbol{c})$ plane. Ligands (in gray) are shown for the LS structure only. b) XRD measurement on a single crystal: temperature evolution of the unit cell volume ( $\mathrm{T}$

6 decreasing, plain black triangles, T increasing, open circles) for extraction of thermal expansion 7 parameters of the HS (red dotted line) and LS (blue dotted line) unit cell (SI Method,

8 [Tissot2011]). Vertical black lines represent transition temperatures for $\mathrm{T}$ decreasing $\left(\mathrm{T}_{\downarrow} \mathrm{SC}^{\mathrm{SC}}\right.$ 9 and $\mathrm{T}$ increasing $\left(\mathrm{T}_{\uparrow}^{\mathrm{SC}}\right)$. c) to $\left.\mathrm{f}\right)$ Synchrotron $\mathrm{x}$-ray powder diffraction on micro-crystals measured at ESRF, ID9 beamline: c) Diffraction image measured at $\mathrm{T}=293 \mathrm{~K}$. d) 1D powder
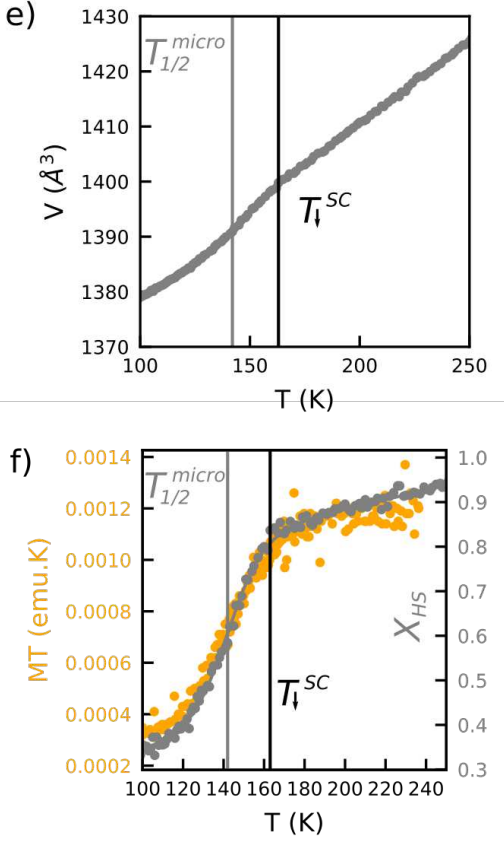
1 measured from $\mathrm{T}=293 \mathrm{~K}$ to $\mathrm{T}=99 \mathrm{~K}$ (upon cooling). e) and $\mathrm{f}$ ) Temperature evolution of the 2 mean unit cell volume as extracted from the powder pattern refinement. e) In yellow: 3 temperature evolution of the magnetic moment-temperature product $M T$ as extracted from 4 SQUID measurements; in grey: evolution of the HS fraction as extracted from the powder 5 pattern refinement. Gray solid line is a guide for the eyes. b), e) and f): The vertical black and 6 gray lines indicate transition temperatures $\mathrm{T}_{\downarrow} \mathrm{SC}$ and $\mathrm{T}_{1 / 2}$ micro in the single crystal and small 7 crystallites, respectively. 
a)

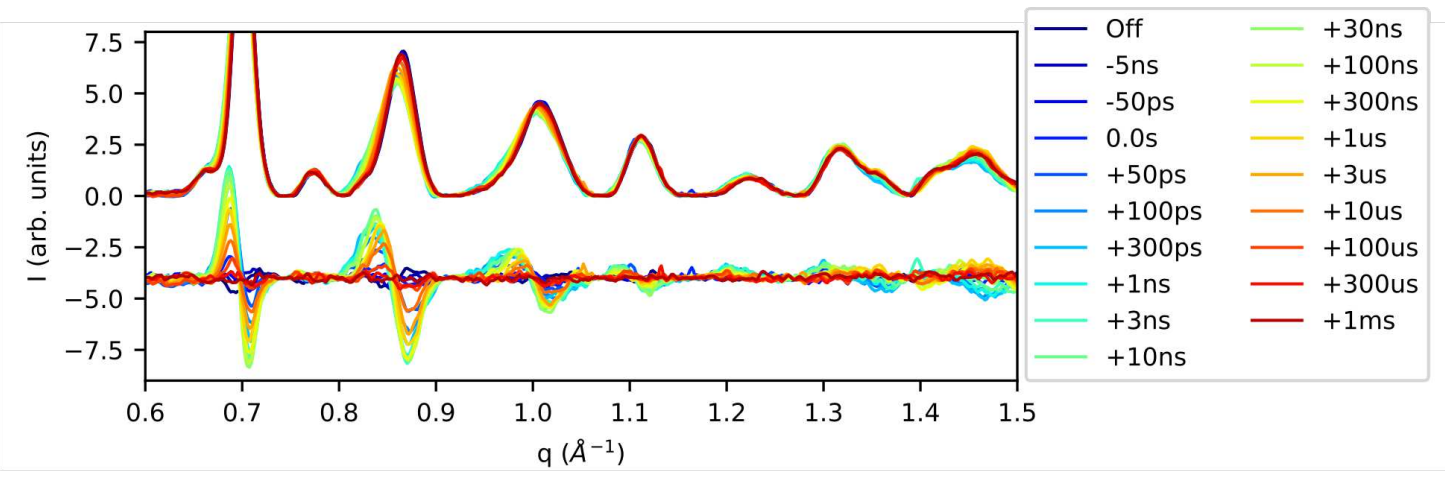

b)

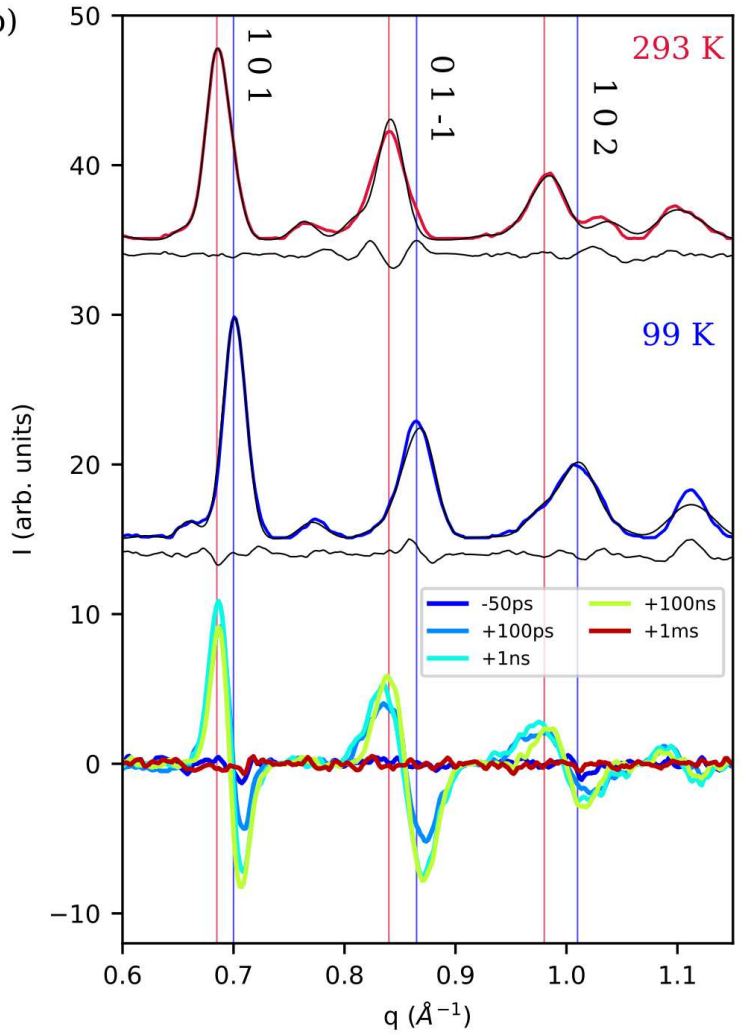

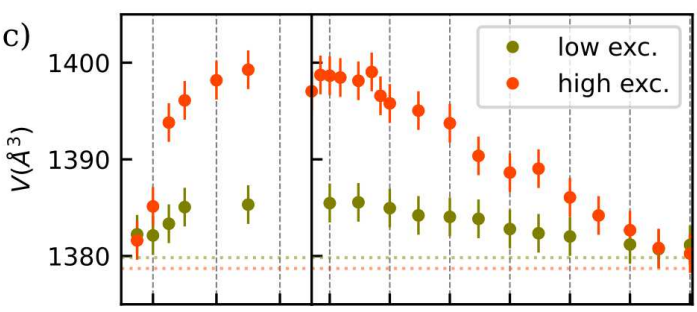
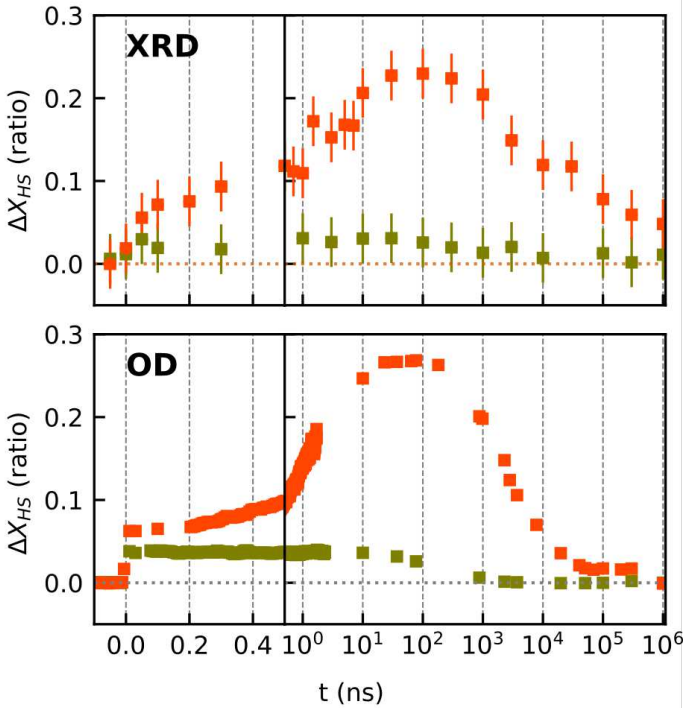

2 Figure 2. a) TR powder patterns and corresponding difference patterns measured under high

3 fluence $380 \mu \mathrm{J} / \mathrm{mm}^{2}$ (multiplied by 2 and shifted by 4 for clarity). Blue to red colors correspond

4 to increasing time delay from negative to positive (detailed delays in Methods). b) Integrated

5 pattern of crystalline film. Top and middle: Steady state integrated patterns measured at $\mathrm{T}=$ $6293 \mathrm{~K}$ (HS state, in red) and T = $99 \mathrm{~K}$ (in blue), offset for clarity by arbitrary values. Black

7 lines are the result of powder pattern refinements (see Methods) and grey lines correspond to

8 the residual (difference between experimental and refined patterns). Bottom: Time-resolved

9 differential pattern measured at $99 \mathrm{~K}$ from -50 ps to $1 \mathrm{~ms}$ after photo-excitation under high 
1 fluence $380 \mu \mathrm{J} / \mathrm{mm}^{2}$ (multiplied by 4 for clarity), showing the characteristic shape for volume

2 expansion (peak shifts towards lower q values). Red and blue vertical lines indicate the position

3 of major HS and LS Bragg peaks, respectively. c) Time evolution of the volume (top) and

4 relative HS fraction (middle) as extracted from powder pattern refinement. Bottom: time

5 evolution of the relative photo-induced HS fraction extracted from optical spectroscopy

6 (adapted from [Bertoni2016a]). In orange: high excitation density $\left(380 \mu \mathrm{J} / \mathrm{mm}^{2}\right.$ and 410

$7 \mu \mathrm{J} / \mathrm{mm}^{2}$ for XRD and OD measurements, respectively), in dark green: low excitation density

$8 \quad\left(60 \mu \mathrm{J} / \mathrm{mm}^{2}\right.$ and $100 \mu \mathrm{J} / \mathrm{mm}^{2}$ for XRD and OD measurements, respectively). 
a)

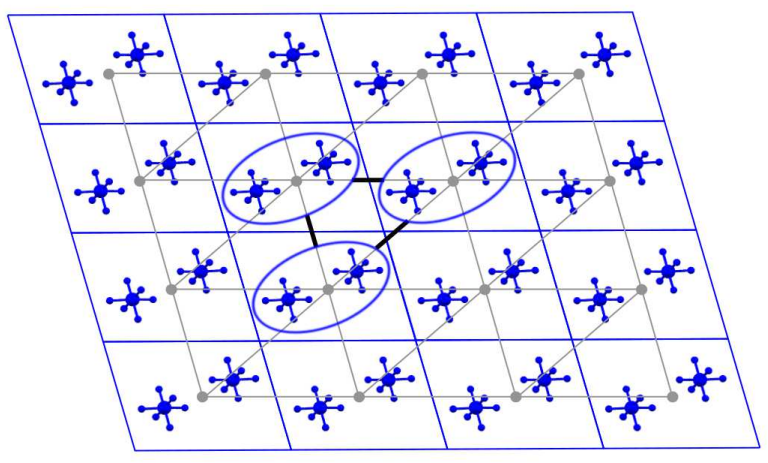

b)

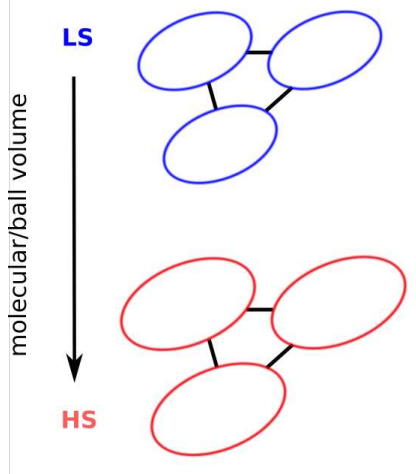

c)

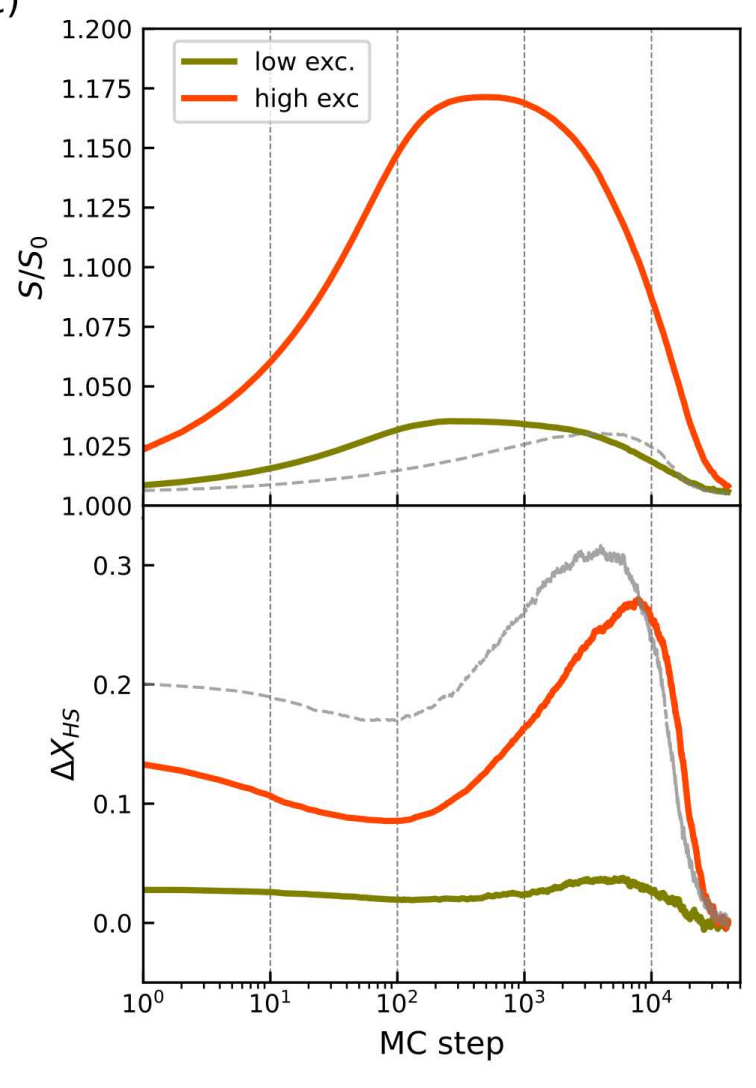

2 Figure 3. a) $\left[\mathrm{Fe}^{\mathrm{III}}(3-\mathrm{MeO}-\mathrm{SalEen})_{2}\right] \mathrm{PF}_{6}$ molecular lattice in $\mathrm{LS}$ state, displayed in the $(\mathrm{a}, \mathrm{c})$

3 plane, for clarity ligands are hidden. The rounded blue contours encircle two molecules per

4 unit cell. In MC simulations, elastic interactions are modelled by springs connecting the unit

5 cells; diagonal springs prevent structural collapse in simulations. b) Schematic representation

6 of ball and spring model accounting for thermal volume expansion. The rounded blue and red

7 contours replicate molecules in LS and HS state, respectively; short black and long magenta

8 sticks replicate the thermal load on the springs c) Simulations of evolution of $X_{\text {HS }}$ (bottom) and

9 of the surface (equivalent to volume in 2D box, top) following high (20\%, orange) and low

10 (5\%, dark green) fractions of switched sites at MC's step zero. Dotted grey lines correspond to previous model, without initial spring length increase. In that case, the increase of HS fraction and the volume were not separated in time. 


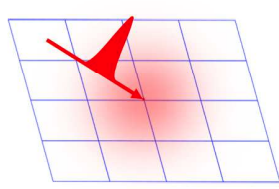

single cristallite (1.5 um)

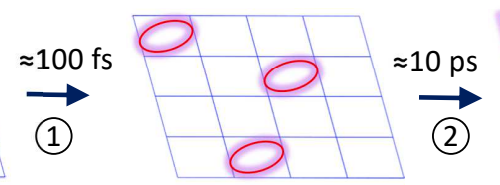

(2)

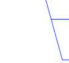

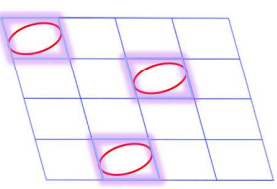

(3) $\mid \approx 100$ ps

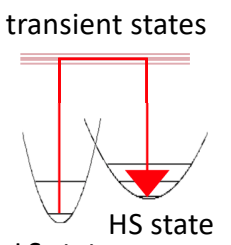

LS state
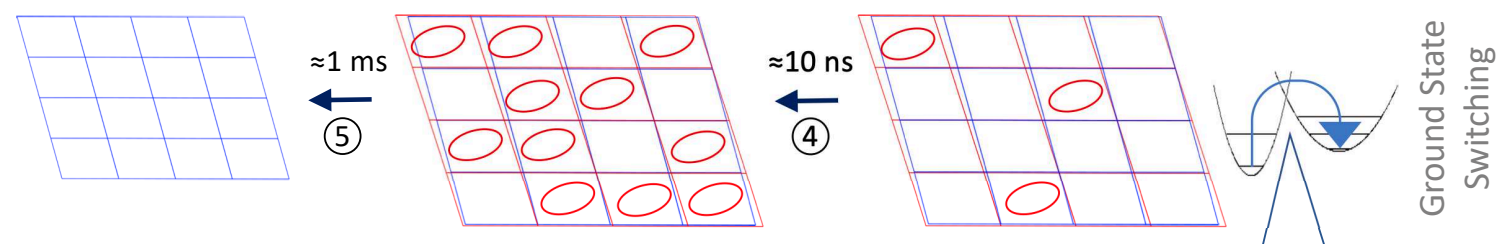

(1) Charge Transfer and cascade through transient states to hot High Spin State

(2) Molecular Vibrational Cooling and Lattice Heating

(3) Lattice Expansion

(4) Arrhenius-type Probability Activation in the Ground State

(5) Recovery of Low Spin Phase and Thermal Equilibrium

2 Figure 4. Photoexcitation cycle of a single crystallite upon spin-crossover. Top: laser induced

3 charge transfer promotes LS molecules (not shown for clarity) residing in LS lattice (blue) to

4 a hot HS state (shape and color coded consistently with figure 3 ) in less than $100 \mathrm{fs}$. Vibrational

5 cooling of hot molecules with LS lattice (color coded consistently with figure 3) takes place on

6 the picosecond time scale. Bottom: volume expansion of heated lattice in 100's ps, and

7 coexistence of LS molecules (not shown) in thermally dilated LS lattice (blue) with photo-

8 switched HS molecules (red) in thermally dilated HS lattice (red). HS and LS lattices

9 superimposed to emphasize their likely coexistence (only average volume measured with

$10 \mathrm{XRD}$ ). Shift of the switching regimes: LS molecules residing in the ground state switch to the

11 HS state according to LS/HS occupancy probabilities modified by lattice expansion; the delay

12 between expansion (300 ps, or less for nano-crystallites) and the switching (ns time-scale), due

13 to incompressible Arrhenius-type kinetics sketched in the insert. 


\section{ACKNOWLEDGEMENTS}

2 ML gratefully acknowledges the Agence Nationale de la Recherche for financial support under 3 grant ANR-16-CE30-0018 ("Elastica"). MLB thanks Amandine Bellec for her assistance in the

4 profilometry measurement. Parts of this research were carried out in the frame of the IM-LED 5 LIA (CNRS).

\section{REFERENCES}

7 Ashiotis2015 G. Ashiotis, A. Deschildre, Z. Nawaz, J. P. Wright, D. Karkoulis, F. E. Picca and 8 J. Kieffer, The fast azimuthal integration Python library: pyFAI, Journal of Applied 9 Crystallography 48, 510 (2015). doi 10.1107/S1600576715004306

Azzolina2019 Azzolina, G., Collet, E., Mariette, C., Cammarata, M., Trzop, E., Sander, M., Levantino, M., Nakagawa, K., Tokoro, H., Ohkoshi, S.-i. and Bertoni, R., Single Laser Shot Photoinduced Phase Transition of Rubidium Manganese Hexacyanoferrate Investigated by Xray Diffraction.

Eur. J. Inorg.

Chem., https://doi.org/10.1002/ejic.201900598

Bargheer2004 M. Bargheer, N. Zhavoronkov, Y. Gritsai, J. C. Woo, D. S. Kim, M. Wörner, and T. Elsässer, Coherent atomic motions in a nanostructure studied by femtosecond X-ray diffraction, Science 306, 5702, 1771-1773 (2004). doi 10.1126/science.1104739

Baum2007, P. Baum, D.-S. Yang, and A. H. Zewail, 4D visualization of transitional structures in phase transformations by electron diffraction, Science 318, 5851, 788-792 (2007). doi $\underline{10.1126 / \text { science. } 1147724}$

Basov2017 D. Basov, R. Averitt and D. Hsieh, Towards properties on demand in quantum materials, Nature Mater 16, 1077-1088 (2017). doi 10.1038/NMAT5017 
1 Bertoni2012 R. Bertoni, M. Lorenc, A. Tissot, M. Servol, M.-L. Boillot and E. Collet,

2 Femtosecond Spin-State Photoswitching of Molecular Nanocrystals Evidenced by Optical

3 Spectroscopy, Angewandte Chemie 51, 7485-7489 (2012). doi 10.1002/anie.201202215

4 Bertoni2016a R. Bertoni, M. Lorenc, H. Cailleau, A. Tissot, J. Laisney, M.-L. Boillot, L.

5 Stoleriu, A. Stancu, C. Enachescu and E. Collet, Elastically driven cooperative response of a 6 molecular material impacted by a laser pulse, Nature Mater. 15, 606-610 (2016). doi $7 \quad \underline{10.1038 / n m a t 4606}$

8 Bertoni2016b R. Bertoni, M. Lorenc, T. Graber, R. Henning, K. Moffat, J.-F. Letard and E.

9 Collet, Cooperative elastic switching vs. laser heating in $\left[\mathrm{Fe}(\mathrm{phen})_{2}(\mathrm{NCS})_{2}\right]$ spin-crossover 10 crystals excited by a laser pulse, CrystEngComm 16, 7269-7275 (2016). doi 10.1039/C6CE00659K

Bertoni2019 R. Bertoni, E. Collet, H. Cailleau, M.-L. Boillot, A. Tissot, J. Laisney, C. Enachescu and M. Lorenc, Temperature dependence of the cooperative out-of-equilibrium elastic switching in a spin-crossover material, Phys. Chem. Chem. Phys. 21, 6606-6612 (2019). doi $10.1039 / \mathrm{C} 8 \mathrm{CP} 07074 \mathrm{~A}$

Bonhommeau2005 S. Bonhommeau, G. Molnár, A. Galet, A. Zwick, J.-A. Real, J. J. McGarvey, and A. Bousseksou, One shot laser pulse induced reversible spin transition in the spin-crossover complex [Fe (C4H4N2) $\{\mathrm{Pt}(\mathrm{CN}) 4\}]$ at room temperature, Angewandte Chemie 117, 26, 4137-4141 (2005). doi 10.1002/anie.200500717

Boukheddaden 2000 K. Boukheddaden, I. Shteto, B. Hôo and F. Varret, Dynamical model for spin-crossover solids. I. Relaxation effects in the mean-field approach, Phys. Rev. B 62, 22, 14796-14805 (2000). doi 10.1103/PhysRevB.62.14796 
1 Braun2007 M. Braun, C. v. Korff Schmising, M. Kiel, N. Zhavoronkov, J. Dreyer, M.

2 Bargheer, T. Elsaesser, C. Root, T. E. Schrader, P. Gilch, W. Zinth, and M. Woerner, Ultrafast

3 Changes of Molecular Crystal Structure Induced by Dipole Solvation, Phys. Rev. Lett. 98, $4 \quad 248301$ (2007). doi 10.1103/PhysRevLett.98.248301

5 Buron2012 M. Buron-Le Cointe, J. Hébert, C. Baldé, N. Moisan, L. Toupet, P. Guionneau, J. 6 F. Létard, E. Freysz, H. Cailleau and E. Collet, Intermolecular control of thermoswitching and 7 photoswitching phenomena in two spin-crossover polymorphs, Phys. Rev. B 85, 064114 8 (2012). doi 10.1103/PhysRevB.85.064114

9 Cailleau2010 H. Cailleau, M. Lorenc, L. Guérin, M. Servol, E. Collet and M. Buron-Le Cointe, 10 Acta Cryst. A66, 2, 189-197 (2010). doi 10.1107/S0108767309051046

Cammarata2009, M. Cammarata, F. Ewald, L. Eybert, W. Reichenbach, M. Wulff, P.

Anfinrud, F. Schotte, Q. Kong, B. Lindenau, J. Rabiger, S. Polachowski, Chopper system for single pulse experiments with synchrotron radiation, Review of Scientific Instruments, 80 015101 (2009). doi 10.1063/1.3036983

Camarata2014 M. Cammarata, R. Bertoni, M. Lorenc, H. Cailleau, S. Di Matteo, C. Mauriac,

S. F. Matar, H. Lemke, M. Chollet, S. Ravy, C. Laulhe, J.-F. Letard and E. Collet, Sequential Activation of Molecular Breathing and Bending during Spin-Crossover Photoswitching Revealed by Femtosecond Optical and X-Ray Absorption Spectroscopy, Phys. Rev. Let. 113, 227402 (2014). doi 10.1103/PhysRevLett.113.227402

Cammarata2017 https://github.com/marcocamma/trx

Chernyshov2004 D. Chernyshov, H.-B. Bürgi, M. Hostettler and K. W. Törnroos, Landau 22 theory for spin transition and ordering phenomena in Fe(II) compounds, Phys. Rev. B 70, 094116 (2004). doi 10.1103/PhysRevB.70.094116 
1 Coelho2018 A.A. Coelho, TOPAS and TOPAS-Academic: an optimization program

2 integrating computer algebra and crystallographic objects written in C++. J. Appl. Cryst., 51, 3210 (2018). doi 10.1107/S1600576718000183

4 Collet2011 E. Collet, N. Moisan, C. Baldé, R. Bertoni, E. Trzop, C. Laulhé, M. Lorenc, M.

5 Servol, H. Cailleau, A. Tissot, M.-L. Boillot, T. Graber, R. Henning, P. Coppens and M.

6 Buron-Le Cointe, Ultrafast spin-state photoswitching in a crystal and slower consecutive

7 processes investigated by femtosecond optical spectroscopy and picosecond X-ray diffraction,

8 Phys. Chem. Chem. Phys., 14, 6192-6199 (2012). doi 10.1039/C2CP23587K

9 Enascescu2017 C. Enachescu, L. Stoleriu, M. Nishino, S. Miyashita, A. Stancu, M. Lorenc, 10 R. Bertoni, H. Cailleau, and E. Collet, Theoretical approach for elastically driven cooperative 11 switching of spin-crossover compounds impacted by an ultrashort laser pulse, Phys. Rev. B 95, 224107 (2017). doi 10.1103/PhysRevB.95.224107

Freyer2013 B. Freyer, F. Zamponi, V. Juvé, J. Stingl, M. Woerner, T. Elsaesser, and M. Chergui, Ultrafast inter-ionic charge transfer of transition-metal complexes mapped by 15 femtosecond X-ray powder diffraction, J. Chem. Phys. 138, 144504 (2013). doi $\underline{10.1063 / 1.4800223}$

Hauser1986 A. Hauser, P. Gütlich and H. Spiering, High-spin $\rightarrow$ low-spin relaxation kinetics and cooperative effects in the $\left[\mathrm{Fe}(\mathrm{ptz})_{6}\left(\mathrm{BF}_{4}\right)_{2}\right.$ and $\left[\mathrm{Zn}_{1 \times x} \mathrm{Fe}_{\mathrm{x}}(\mathrm{ptz})_{6}\right]\left(\mathrm{BF}_{4}\right)_{2}(\mathrm{ptz}=1$-Propyltetrazole $)$ spin-crossover systems. Inorg. Chem. 25, 4245_4248 (1986). doi 10.1021/ic00243a036

Laisney2020 J. Laisney, D. Morineau, C. Enachescu, R. Tanasa, E. Riviere, R. Guillot and M.L. Boillot, Mechanical-tuning of the cooperativity of SC particles via the matrix crystallization and related size effects, Journal of Material Chemistry 8, 7067 (2020). doi 10.1039/d0tc00067a 
1 Landau1980 L. D. Landau and E. M. Lifshitz, Statistical Physics 3 ${ }^{\text {rd }}$ Edition Part 1, Elsevier 2 (1980). https://doi.org/10.1016/C2009-0-24487-4

3 Lorenc2009 M. Lorenc, J. Hebert, N. Moisan, E. Trzop, M. Servol, M. Buron-Le Cointe, H. 4 Cailleau, M.-L. Boillot, E. Pontecorvo, M. Wulff, S. Koshihara and E. Collet, Successive 5 Dynamical Steps of Photoinduced Switching of a Molecular Fe(III) Spin-Crossover Material 6 by Time-Resolved X-Ray Diffraction, Phys. Rev. Lett. 103, 028301 (2009). doi $7 \quad \underline{10.1103 / \text { PhysRevLett.103.028301 }}$

8 Lorenc2012 M. Lorenc, C. Balde, W. Kaszub, A. Tissot, N. Moisan, M. Servol, M. Buron-Le 9 Cointe, H. Cailleau, P. Chasle, P. Czanercki, M.-L. Boillot and E. Collet, Cascading 10 photoinduced, elastic, and thermal switching of spin states triggered by a femtosecond laser 11 pulse in an Fe(III) molecular crystal, Phys. Rev. B 85, 054302 (2012). doi 10.1103/PhysRevB.85.054302

Matsuda2015 O. Matsuda, M. C. Larciprete, R. Li Voti and O. B. Wright, Fundamentals of picosecond laser ultrasonics, Ultrasonics 56, 3-20 (2015). doi 10.1016/j.ultras.2014.06.005

Mariette2021 C. Mariette, M. Lorenc, H. Cailleau, E. Collet, L. Guérin, A. Volte, E. Trzop et al., Strain wave pathway to semiconductor-to-metal transition revealed by time resolved X-ray powder diffraction, Nat. Comm. 12, 1239 (2021). doi 10.1038/s41467-021-21316-y

Morrison2014 V. Morrison, R. P. Chatelain, K. L. Tiwari, A. Hendaoui, A. Bruhács, M. Chaker, and B. J. Siwick, A photoinduced metal-like phase of monoclinic $\mathrm{VO}_{2}$ revealed by ultrafast electron diffraction, Science 346, 6208, 445-448 (2014). doi 10.1126/science.1253779

Nasu1997 K. Nasu K., Multistabilities of the Ground States, Proliferations of Excitons and 
1 and Photo-Induced Structural Phase Transitions. Springer Series in Solid-State Sciences, vol

2 124. Springer, Berlin, Heidelberg (1997). doi 10.1007/978-3-642-60702-8_1

$3 \quad$ Nasu2004 K. Nasu, (ed.) Photoinduced Phase Transitions (World Scientific, 2004). doi $4 \quad \underline{10.1142 / 5476}$

$5 \quad$ Park2017, S. T. Park and R. M. van der Veen, Modeling nonequilibrium dynamics of phase 6 transitions at the nanoscale: Application to spin-crossover, Structural Dynamics 4 (4), 044028 7 (2017). doi $10.1063 / 1.4985058$

8 Parpiiev2017 T. Parpiiev, M. Servol, M. Lorenc, I. Chaban, R. Lefort, E. Collet, H. Cailleau, 9 P. Ruello, N. Daro, G. Chastanet, and T. Pezeril, Ultrafast non-thermal laser excitation of 10 gigahertz longitudinal and shear acoustic waves in spin-crossover molecular crystals [Fe(PM11 AzA) $\left.)_{2}(\mathrm{NCS})_{2}\right]$, Appl. Phys. Lett. 111, 151901 (2017). doi: 10.1063/1.4996538

Pawley1981 Pawley, G. S., Unit-cell refinement from powder diffraction scans, Journal of Applied Crystallography, 14, 6, 357-361 (1981).doi 10.1107/S0021889881009618

Rat2017 S. Rat, K. Ridier, L. Vendier, G. Molnár, L. Salmon, and A. Bousseksou,

Solvatomorphism and structural-spin crossover property relationship in bis[hydrotris(1\{,\}2\{,\}4-triazol-1-yl)borate]iron(ii), CrystEngComm 19, 24, 3271-3280 (2017).doi 10.1039/C7CE00741H

Rene2017 L. P. René de Cotret and B. J. Siwick, A general method for baseline-removal in ultrafast electron powder diffraction data using the dual-tree complex wavelet transform, Struct. Dyn. 4 (2017). doi 10.1063/1.4972518

Ridier2019 K. Ridier, A.-C. Bas, V. Shalabaeva, W. Nicolazzi, L. Salmon, G. Molnár, A. 
1 Switching Dynamics of Spin-Crossover Thin Films Photoexcited by a Femtosecond Laser

2 Pulse. Adv. Mater. 2019, 31, 1901361 (2019). doi 10.1002/adma.201901361

3 Rowles2017 M. R. Rowles and C. E. Buckley, Aberration corrections for non-Bragg-Brentano

4 diffraction geometries, J. Appl. Cryst. 50, 240-251 (2017). doi 10.1107/S1600576717000085

5 Ruello2015 P. Ruello and V. E. Gusev, Physical mechanisms of coherent acoustic phonon 6 generation by ultrafast laser action, Ultrasonics 56, 21-35 (2015). doi $7 \quad$ 10.1016/j.ultras.2014.06.004

8 Sagar2016, D. M. Sagar, F. G. Baddour, P. Konold, J. Ullom, D. A. Ruddy, J. C. Johnson and

9 R. Jimenez, Femtosecond Measurements Of Size-Dependent Spin Crossover In $10 \mathrm{FeII}(\mathrm{pyz}) \mathrm{Pt}(\mathrm{CN})_{4}$ Nanocrystals. The journal of physical chemistry letters, 7(1), 148-153 (2016). 11 doi 10.1021/acs.jpclett.5b02435

12 Schick2014 D. Schick, M. Herzog, A. Bojahr, W. Leitenberger, A. Hertwig, R. Shayduk and 13 M. Bargheer, Ultrafast lattice response of photoexcited thin films studied by X-ray diffraction, 14 Struct. Dyn. 1,064501 (2014). doi 10.1063/1.4901228

Thomsen1986 C. Thomsen, H. T. Grahn, H. J. Maris and J. Tauc, Surface generation and detection of phonons by picosecond light pulses, Phys. Rev. B 34, 6, 4129-4138 (1986). doi $\underline{10.1103 / \text { PhysRevB.34.4129 }}$

Tissot2011 A. Tissot, R. Bertoni, E. Collet, L. Toupet and M.-L. Boillot, The cooperative spinstate transition of an iron(iii) compound $\left[\mathrm{Fe}^{\mathrm{m}}(3-\mathrm{MeO}-\mathrm{SalEen})_{2}\right] \mathrm{PF}_{6}$ : thermal- vs. ultra-fast photo-switching, J. Mater. Chem. 21, 18347-18353 (2011). doi 10.1039/c1jm14163e

Tissot2012 A. Tissot, L. Rechignat, A. Bousseksou and M.-L. Boillot, Micro- and nanocrystals of the iron(iii) spin-transition material $\left[\mathrm{Fe}^{\mathrm{m}}(3-\mathrm{MeO}-\mathrm{SalEen})_{2}\right] \mathrm{PF}_{6}$, J. Mater. Chem. 22, 34113419 (2012). doi 10.1039/C2JM14913C 
1 VanderVeen2013a, R.-M. Van der Veen, A. Tissot, A. Hauser and A.-H. Zewail, Unusual

2 molecular material formed through irreversible transformation and revealed by 4D electron

3 microscopy, Physical Chemistry Chemical Physics 15, 20, 7831-7838 (2013). doi

$4 \quad \underline{0.1039 / \mathrm{c} 3 \operatorname{cp} 51011 \mathrm{e}}$

5 VanderVeen2013b, R.-M. Van Der Veen, O.-H. Kwon, A. Tissot, A. Hauser and A.-H. Zewail,

6 Single-nanoparticle phase transitions visualized by four-dimensional electron microscopy,

$7 \quad$ Nature Chemistry 5 (5), 395 (2013). doi 10.1038/nchem.1622

8 Wright1994 O. B. Wright, Ultrafast nonequilibrium stress generation in gold and silver, Phys.

9 Rev. B 49, 14, 9985-9988 (1994). doi 10.1103/PhysRevB.49.9985

10 Zhang2014 J. Zhang and R. D. Averitt, Dynamics and Control in Complex Transition Metal

11 Oxides, Annu. Rev. Mater. Res. 44, 19 (2014). doi 10.1146/annurev-matsci-070813-113258 


\section{Supplementary Files}

This is a list of supplementary files associated with this preprint. Click to download.

- DynamicallimitsVolteSI.pdf 